\title{
Frequency-Warped Filter Banks and Wavelet Transforms: A Discrete-Time Approach via Laguerre Expansion
}

\author{
Gianpaolo Evangelista, Member, IEEE, and Sergio Cavaliere
}

\begin{abstract}
In this paper, we introduce a new generation of perfect-reconstruction filter banks that can be obtained from classical critically sampled filter banks by means of frequency transformations.

The novel filters are Laguerre type IIR filters that can be directly derived and designed from ordinary orthogonal or biorthogonal filter banks. Generalized downsampling and upsampling operators based on dispersive delay lines are the building blocks of our structures.

By iterating the filter banks, we construct new orthogonal and complete sets of wavelets whose passbands are not octave spaced and may be designed by selecting a single parameter.
\end{abstract}

\section{INTRODUCTION}

$\mathbf{T}$ THE THEORY and application of multirate filter banks has received considerable interest in the last decade, and the relationships with orthogonal and biorthogonal transforms, such as the wavelet transform, wavelet packets, and lapped orthogonal transforms have been exploited to provide more insight in the topic and new applications. The limitations of the conventional critically sampled filter banks often lie in the integer or, at best, rational sampling rates achieved at the outputs of the analysis filters. These rates determine strict constraints on the passbands, conditioning the tiling of the time-frequency plane that is obtained.

As suggested in recent papers [6], [7], unitary equivalence may be exploited in order to design new transforms, leading to representations that can be adapted to signals. Examples of these operations are time and frequency warping. However, the setting of [7] is very general and mostly refers to continuoustime signal processing.

In this paper, we propose novel discrete-time orthogonal and biorthogonal filter bank structures based on multirate Laguerre sequence expansions. These structures are derived by introducing generalized downsampling and upsampling operations characterized by frequency-dependent sampling rates. Central to the implementation of these resamplers are (adopting a terminology borrowed from wave propagation) the lossless dispersive delay lines based on allpass filters. In these lines, different frequency components of the signal travel with different speed.

Manuscript received June 20, 1996; revised March 9, 1998. The associate editor coordinating the review of this paper and approving it for publication was Dr. Truong Q. Nguyen.

The authors are with the Department of Physical Sciences, University "Federico II" Naples, Napoli, Italy (e-mail: evangelista@na.infn.it).

Publisher Item Identifier S 1053-587X(98)07066-4
As we will show, the Laguerre expansion can be used to form new filter bank structures: either parallel or iterated. In particular, we define new frequency-warped wavelets. Unlike the ordinary dyadic wavelets, the frequency bands of warped wavelets are not organized in octaves. Rather, the cutoff frequencies may be chosen by varying the so-called Laguerre parameter. In several applications, such as audio coding, analysis, synthesis, and denoising, the choice of the proper tiling of the time-frequency plane may dramatically improve performance. By adapting the frequency bands to the signal, we can enhance energy compaction and improve the coding efficiency. The Laguerre parameter in the frequency-warped filter banks or wavelets provides an additional degree of freedom in the choice of the frequency bands. For example, an entire class of nonuniform filter banks may be derived from uniform filter banks simply by changing the Laguerre parameter.

The computational complexity of the new class of filter banks is higher than that of conventional ones, and only approximated reconstruction is possible in finite time with an input of finite duration.

A review of Laguerre sequences and related expansions is presented in Section II. There, the relationships between frequency warping and Laguerre expansions are pointed out. In Section III, we combine Laguerre expansions with filter banks, obtaining frequency-warped filter bank structures. These structures are based on generalized resampling operators described in Section IV. In Section V, we apply our concepts to the wavelet transform via iterated filter banks, defining discretetime frequency-warped wavelets, and compare the complexities of the computational structures. Finally, in Section VI, we draw our conclusions.

\section{LAGUerRe SEQUence EXPANSIONS}

\section{A. Properties of Laguerre Sequences}

The Laguerre sequences [1]

$$
\begin{aligned}
& \lambda_{r}(k ; b)= \sqrt{1-b^{2}} \sum_{m=0}^{\min (r, k)}(-1)^{m+r} \\
& \times \frac{(k+r-m) !}{m !(k-m) !(r-m) !} b^{r+k-2 m} \\
& r, k=0,1, \ldots .
\end{aligned}
$$




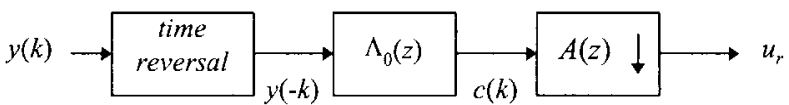

Fig. 1. Structure for computing the Laguerre coefficients.

form a complete orthonormal set in the space $l^{2}$ of finiteenergy causal sequences for any fixed value of the parameter $b$ such that $-1<b<1$. They are obtained by performing Gram-Schmidt orthogonalization on the sequences $h_{r}(k)=$ $k^{r} b^{k}$ [8]. Since the $Z$ transform of the order- $r$ sequence [9] is

$$
\Lambda_{r}(z)=\sqrt{1-b^{2}} \frac{\left(z^{-1}-b\right)^{r}}{\left(1-b z^{-1}\right)^{r+1}}
$$

the Laguerre sequences satisfy the following $Z$-transform recurrence ${ }^{1}$ :

$$
\Lambda_{r+1}(z)=A(z) \Lambda_{r}(z)=A(z)^{r+1} \Lambda_{0}(z), \quad r=0,1, \ldots
$$

where

$$
A(z)=\frac{z^{-1}-b}{1-b z^{-1}}
$$

is the transfer function of a real, stable, and causal first-order allpass filter. On the unit circle, we have $A\left(e^{j \omega}\right)=e^{-j \theta(\omega)}$, where

$$
\theta(\omega)=-\arg A\left(e^{j \omega}\right)=\omega+2 \tan ^{-1}\left(\frac{b \sin \omega}{1-b \cos \omega}\right)
$$

Consider a discrete-time causal signal $y(k)$ and its expansion in terms of Laguerre sequences

$$
y(k)=\sum_{r=0}^{\infty} u_{r} \lambda_{r}(k ; b)
$$

where

$$
u_{r}=\sum_{k=0}^{\infty} y(k) \lambda_{r}(k ; b)
$$

In order to simplify our notation, in the expression of the Laguerre coefficients $u_{r}$ and in most of the subsequent equations, we will omit the dependency on $b$ unless it is specifically relevant.

Equation (7) may be interpreted as a time-varying filtering operation on $y(k)$ with impulse response $h(r, k)=\lambda_{r}(k ; b)$, which, according to the time domain counterpart of (3), has the remarkable property $h(r+1, k)=h(r, k) * \alpha(k)$, where $\alpha(k)$ is the impulse response of the allpass section $A(z)$. An iterative, noncausal scheme to compute the Laguerre coefficients based on (7) and recurrence (3) is given by the diagram shown in Fig. 1. There, the input signal is time-reversed and convolved with the zero-order Laguerre sequence, whose $Z$ transform is

$$
\Lambda_{0}(z)=\frac{\sqrt{1-b^{2}}}{1-b z^{-1}}
$$

\footnotetext{
${ }^{1}$ Throughout this paper, we will denote by $f(z)^{r}$ the $r$ th power of $f(z)$. We prefer this notation to the standard $f^{r}(z)$ both to avoid confusion between inverse and reciprocal when $r=-1$ and to point out the substitution of the delay $z^{-r}$ with the frequency-dependent delay $A(z)^{r}$.
}

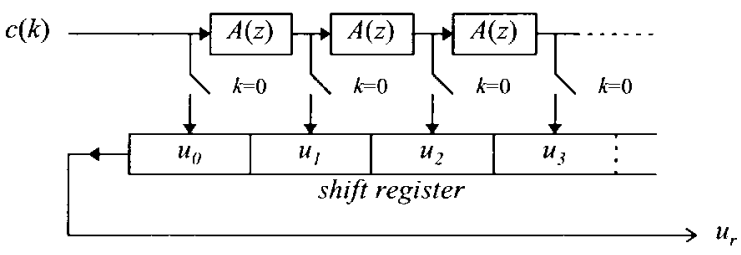

(a)

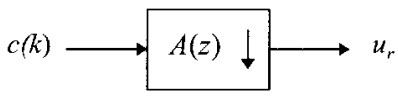

(b)

Fig. 2. (a) Diagram of the switched dispersive delay line needed to compute the Laguerre coefficients. (b) Its equivalent schematic symbol.

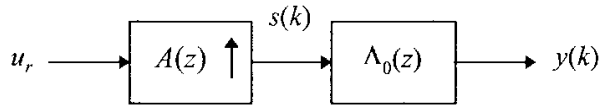

Fig. 3. Structure for reconstructing a signal from its Laguerre coefficients.

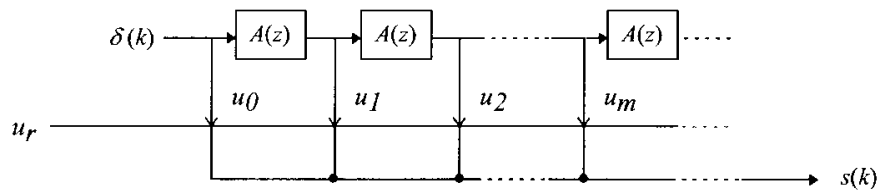

(a)

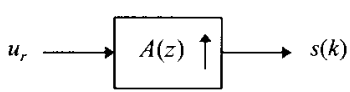

(b)

Fig. 4. (a) Diagram of the tapped dispersive delay line needed to reconstruct the signal from its Laguerre coefficients. (b) Its equivalent schematic symbol.

with

$$
\Lambda_{0}(z)^{2}=\frac{d}{d z^{-1}} A(z)
$$

This filter is lowpass for $b>0$ and highpass for $b<0$. The signal is then fed to the dispersive delay-line shown in Fig. 2(a) and denoted by the symbol shown in Fig. 2(b). The switches of the network of Fig. 2(a) close at time $k=$ 0 , loading, at that instant, the shift register, whose output provides, at subsequent time intervals, the ordered sequence of Laguerre coefficients. Since the structure is anticausal and the filters involved are IIR, the Laguerre coefficients can be computed in finite time only if the input is time limited. If $y(k) \neq 0$ only for $k=0,1, \ldots, N$, we can perform a timereversal operation within a finite delay, obtaining the sequence $x(k)=y(N-k)$, which is then fed to the $\Lambda_{0}(z)$ filter and then input to a network similar to that shown in Fig. 2(a). In this case, however, the switches are to be closed at time $k=N$. Furthermore, it should be clear that only a finite number of Laguerre coefficients can be computed in finite time.

Since the Laguerre sequences form a complete set, the signal may be reconstructed from its Laguerre coefficients. The diagram of the synthesis structure is given in Fig. 3. There, the "input" sequence $u_{k}$ forms the weights of the Laguerre synthesis network detailed in Fig. 4(a) and denoted by the symbol shown in Fig. 4(b). The output of this network is then 
filtered by $\Lambda_{0}(z)$, retrieving the original signal. It should be pointed out that the Laguerre synthesis network is an infinitelength dispersive tapped delay line excited by the unit pulse sequence $\delta(k)$. The sequence $u_{k}$ is not an input in the classical sense. Rather, the entire sequence must be available at time $k=0$ in order to perform the required products. In a noncausal structure, we may load these coefficients in an infinitely long shift register. At the time origin, the register is fully loaded, and the content of its cells will be available to the multipliers.

An alternate synthesis structure may be obtained from the following remarks. By defining the transposed Laguerre sequences $\lambda_{k}^{+}(r ; b) \equiv \lambda_{r}(k ; b)$, (6) may be put in the same form as (7)

$$
y(k)=\sum_{r=0}^{\infty} u_{r} \lambda_{k}^{+}(r ; b) .
$$

As can be seen from (1), the indices $r$ and $k$ play a symmetric role, except for the signature factor $(-1)^{m+r}$, and we can easily verify that $\lambda_{k}^{+}(r ; b) \equiv \lambda_{k}(r ;-b)$. Therefore, Laguerre synthesis may also be computed by means of the analysis structure, driven by the expansion coefficients $u_{r}$, with $b$ replaced by $-b$.

In practice, the synthesis network must be truncated to a finite number of elements, and only a finite number of coefficients may be computed. Moreover, we need to assume that the input has finite duration. In that case, the noncausal structure may be transformed in a causal structure, computing the output within a finite delay. The signal is approximated by a truncated Laguerre expansion, and an estimate of the number of coefficients required may be given in terms of the group delay of the filter chain in Figs. 1 and 2 . The $M$ th coefficient is computed by the filter $\Lambda_{0}(z)$ cascaded by $M-1$ allpass filters. Thus, the total group delay is the sum

$$
\tau_{G, M-1}(\omega)=\tau_{G, 0}(\omega)+(M-1) \tau_{G, 1}(\omega)
$$

where

$$
\tau_{G, 1}(\omega)=\frac{d \theta}{d \omega}=\frac{1-b^{2}}{1-2 b \cos \omega+b^{2}}
$$

is the group delay of each allpass filter and

$$
\tau_{G, 0}(\omega)=\frac{\tau_{G, 1}(\omega)-1}{2}
$$

is the group delay of $\Lambda_{0}(z)$.

Therefore

$$
\begin{aligned}
\tau_{G, M-1}(\omega) & =(M-1 / 2) \tau_{G, 1}(\omega)-1 / 2 \\
& =\frac{M\left(1-b^{2}\right)+b \cos \omega-1}{1-2 b \cos \omega+b^{2}} .
\end{aligned}
$$

In the worst case of full bandwidth signals, the minimum total group delay is attained at $\omega=\pi$ if $b>0$ or at $\omega=0$ if $b>0$; hence

$$
\tau_{\min , M-1}=\min _{\omega} \tau_{G, M-1}(\omega)=\frac{M(1-|b|)-1}{1+|b|} .
$$

If the duration of the input signal is $D$ samples, it is clear that at the time instant $k=0$, when the switches of the
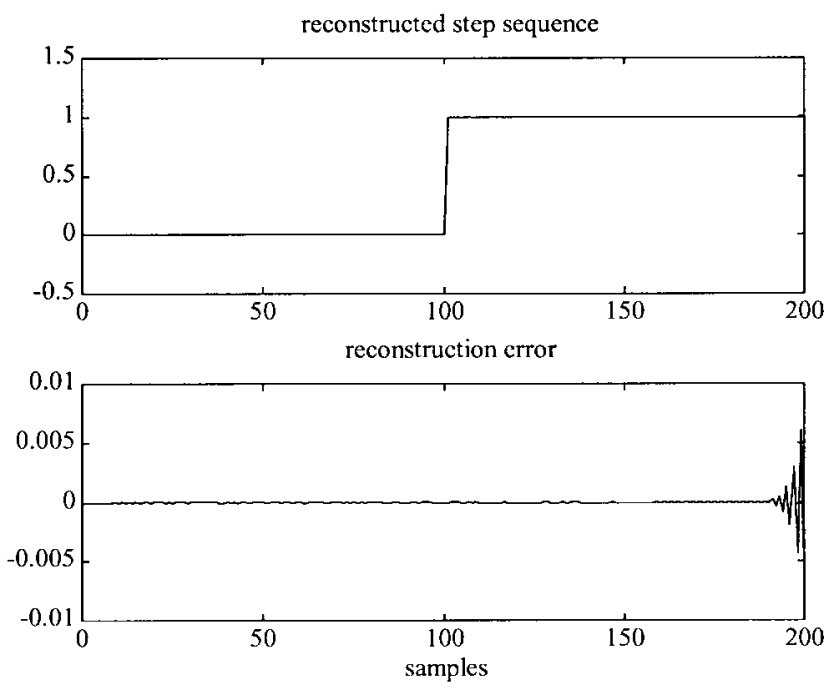

Fig. 5. Truncated Laguerre expansion: Reconstructed step sequence and error with 600 terms, where $b=0.5$.

network in Fig. 2 are closed, the output of the $M+1$ and subsequent sections will be approximately zero, provided that $\tau_{\min , M-1} \geq D-1$, obtaining the following lower bound on the number $M$ of Laguerre coefficients that must be computed

$$
M \geq M_{\min } \equiv \frac{(D-1)(1+|b|)+1}{1-|b|} \approx \frac{D(1+|b|)}{1-|b|} .
$$

Notice that the ratio $M_{\min } / D$ grows with $|b|$, i.e., when the filter pole moves toward the unit circle, attaining its minimum value when $b=0$, i.e., when the allpass filters revert to unit delays, in which case, obviously, the bound is exactly met: $M=M_{\min }=D$. As a numerical example, for $b=0.5$, about three Laguerre coefficients per input samples must be computed. The approximation to 600 coefficients of a 200sample step sequence is reported in Fig. 5, together with the truncation error, which turns out to be less than $10^{-2}$.

In Fig. 6, a plot of the maximum absolute value of the first 2000 Laguerre sequences in an analysis interval of $D=100$ samples is shown. Each curve corresponding to a distinct value of $b$ has a "knee" centered on the respective value of $M_{\text {min }}$, as estimated by means of (10). The values of the higher order Laguerre sequences abruptly drop below significant level and may be disregarded, thereby showing the consistency of (10).

Due to the delay of the Laguerre sequences, the error obtained by truncating the Laguerre series is larger at the end of the analysis interval. Since the group delay depends on frequency, the spectrum of the error depends on the sign of $b$. For negative values of $b$, the error is larger at lower frequencies and vice-versa for positive $b$. The error is larger at higher frequencies.

\section{B. Frequency Warping via Laguerre Expansion}

Next, we will show that the Laguerre expansions are related to a special case of frequency warping [4], [5]. $Z$ transforming both sides of the Laguerre expansion of the signal $y(k)$ [see 


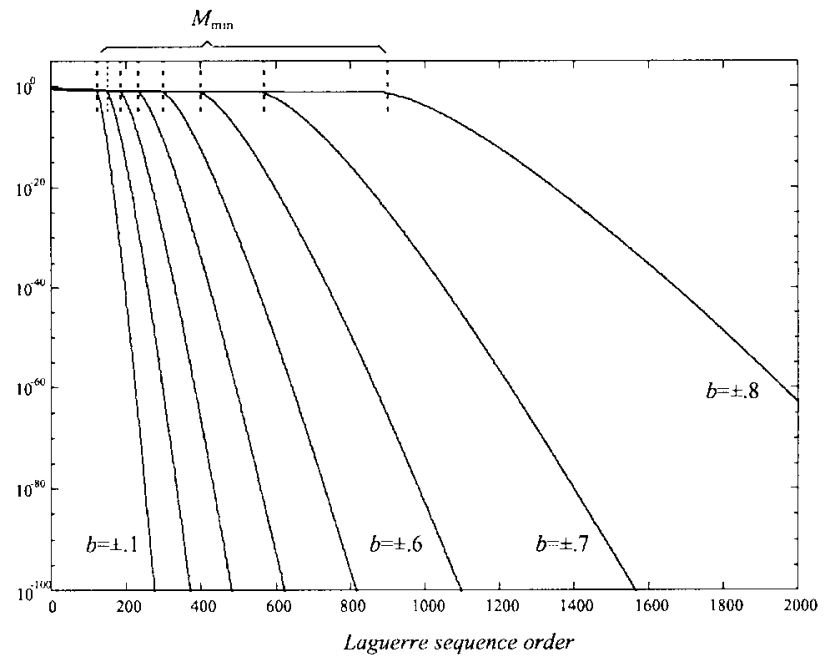

Fig. 6. Diagram of the absolute maximum value of the first $D=100$ samples of Laguerre sequences up to order 2000 for several values of the parameter $b$. Dashed lines specify $M_{\min }$ corresponding to distinct values of $b$.

(6)], we obtain

$$
Y(z)=\sum_{r=0}^{\infty} u_{r} \Lambda_{r}(z)
$$

Substituting in this expression recurrence (3) and factoring out $\Lambda_{0}(z)$, we have

$$
Y(z)=\Lambda_{0}(z) \sum_{r=0}^{\infty} u_{r} A(z)^{r}=\Lambda_{0}(z) U\left(A(z)^{-1}\right) .
$$

As stated in (4), the transfer function $A(z)$ is allpass; therefore, $A\left(e^{j \omega}\right)=e^{-j \theta(\omega)}$ on the unit circle, and

$$
Y\left(e^{j \omega}\right)=\Lambda_{0}\left(e^{j \omega}\right) U\left(e^{j \theta(\omega)}\right)
$$

where

$$
U\left(e^{j \theta(\omega)}\right)=\sum_{r=0}^{\infty} u_{r} e^{-j r \theta(\omega)}
$$

with $\theta(\omega)$ given in (5).

Equation (13) has a straightforward interpretation. The frequency spectrum of the sequence of Laguerre coefficients $u_{r}$ is a frequency-warped version of the original frequency spectrum $Y\left(e^{j \omega}\right)$ weighted by $\Lambda_{0}\left(e^{j \omega}\right)^{-1}$, which is the frequency response of an FIR filter. The warping $\Omega=\theta(\omega)$ of the frequency axis is controlled by the parameter $b$ of the allpass filter (4). A family of curves showing the features of the frequency map for several values of $b$ is reported in Fig. 7. The $Z$-domain form of this map is $z^{-1} \rightarrow A(z)$, which is a one-toone form and maps the unit disc onto itself. It is the unique real rational map with unitary modulus on the unit circle (allpass) [4], [5]. It is easy to show by direct substitution that

$$
A\left(A^{+}(z)^{-1}\right)=A^{+}\left(A(z)^{-1}\right)=z^{-1}
$$

where

$$
A^{+}(z)=\frac{z^{-1}+b}{1+b z^{-1}}
$$

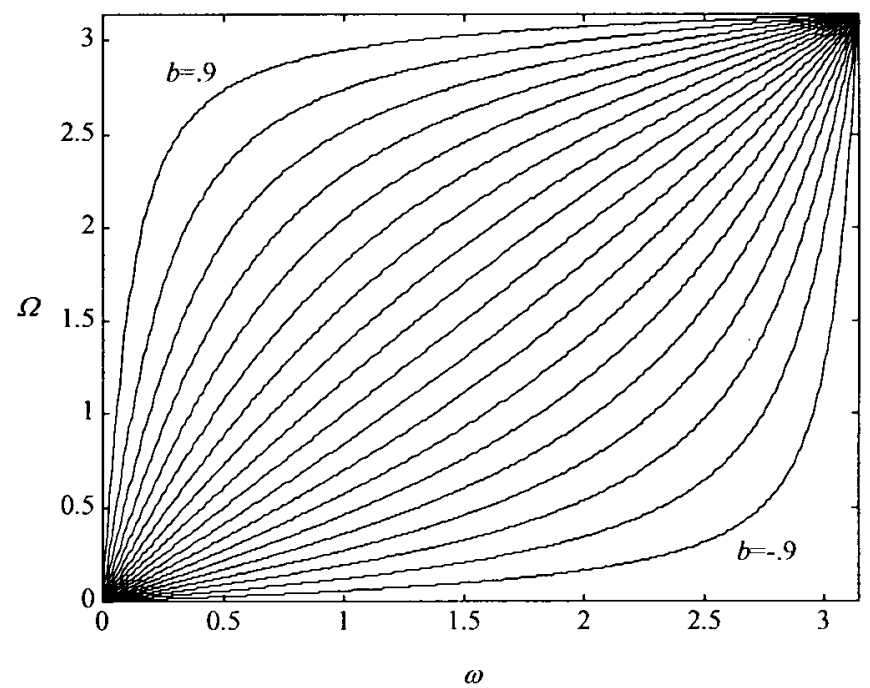

Fig. 7. Family of frequency warping curves with the Laguerre parameter $b$ ranging from -0.9 to 0.9 in increments of 0.1 .

Thus, the inverse mapping is $z^{-1} \rightarrow A^{+}(z)$ and the unwarping map $\omega=\theta^{-1}(\Omega)$ is obtained from (5) by replacing $b$ with $-b$ and $\omega$ with $\Omega$.

An alternate expression for $U(z)$ may be derived in terms of the transposed Laguerre sequences $\lambda_{k}^{+}(r ; b) \equiv \lambda_{r}(k ; b)$, whose $Z$ transforms are $\Lambda_{k}^{+}(z) . Z$ transforming (7) and exploiting recurrence (3) written for $\Lambda_{k}^{+}(z)$ yields

$$
U(z)=\Lambda_{0}^{+}(z) Y\left(A^{+}(z)^{-1}\right)
$$

where $\Lambda_{0}^{+}(z)=\frac{\sqrt{1-b^{2}}}{1+b z^{-1}}$. Since $\Lambda_{0}^{+}\left(A(z)^{-1}\right)=\Lambda_{0}(z)^{-1}$, by applying (15), we can show the equivalence of (12) and (16). The latter shows that $Y(z)$ is a frequency unwarped version of $U(z)$ weighted by the FIR frequency response $\Lambda_{0}^{+}(z)^{-1}$.

The frequency warping method illustrated in [5] is obtained by means of biorthogonal sequences $\psi_{k}(n)$ and $\phi_{k}(n)$ such that

$$
\Psi_{k}(z)=A(z)^{k}, \quad k=0,1 \ldots
$$

are the $Z$ transforms of the synthesis sequences, and

$$
\phi_{k}(n)= \begin{cases}b^{n}, & k=0 \\ \frac{n}{k} \psi_{k}(n), & k=1,2, \ldots\end{cases}
$$

are the analysis sequences. The relationships between the biorthogonal frequency warping set and Laguerre sequences, based on the same allpass transfer function $A(z)$, are easily found in the $Z$-transform domain as

$$
\begin{aligned}
\Psi_{k}(z) & =\frac{\Lambda_{k}(z)}{\Lambda_{0}(z)} \\
\Phi_{0}(z) & =\frac{1}{\sqrt{1-b^{2}}} \Lambda_{0}(z) \\
\frac{\Phi_{k+1}(z)}{z^{-1} \Phi_{0}(z)} & =\sqrt{1-b^{2}} \Lambda_{k}(z), \quad k=0,1, \ldots
\end{aligned}
$$

Due to orthogonality, the frequency warping obtained by means of Laguerre analysis preserves the total energy by weighting the original frequency spectrum with $\Lambda_{0}\left(e^{j \omega}\right)^{-1}$. The same is not true for the biorthogonal frequency warping set, which purely warps the frequency axis. 


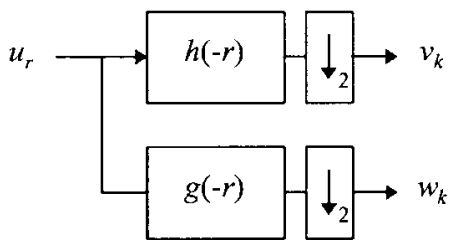

Fig. 8. Two-band critically sampled analysis filter bank.

\section{LAGUERRE DOMAIN FILTER BANKS}

In this section, we combine critically sampled filter banks with Laguerre expansions. This leads to the definition of filter banks in the Laguerre domain. As remarked in Section II$\mathrm{B}$, the sequence of Laguerre coefficients $u_{r}$ constitutes a frequency-warped version of the input signal. This sequence can be directly fed into a perfect-reconstruction filter bank. We will show that Laguerre expansion cascaded by a multirate filter bank is equivalent to a filter bank embedding the frequency warping operation both in the component filters and the resampling operators. The definition of discrete-time frequency-warped wavelets is based on this property.

In order to simplify our notation, we shall confine ourselves to considering the two-band real orthogonal filter bank shown in Fig. 8, which is characterized by two QMF impulse responses $h$ (lowpass) and $g$ (highpass), which satisfy the aliasing cancellation condition [12], [13]

$$
\mathbf{T}(z) \tilde{\mathbf{T}}(z)=2 \mathbf{I}
$$

where

$$
\mathbf{T}(z) \equiv\left[\begin{array}{cc}
H(z) & G(z) \\
H(-z) & G(-z)
\end{array}\right]
$$

is the ac matrix, and $\tilde{\mathbf{T}}(z) \equiv \mathbf{T}^{t}\left(z^{-1}\right)$ is its transpose evaluated in $z^{-1}$. Assuming anticausal analysis filters, the outputs of the downsamplers, respectively, are

$$
v_{k}=\sum_{r} h(r-2 k) u_{r}
$$

and

$$
w_{k}=\sum_{r} g(r-2 k) u_{r}
$$

Substituting (7) in the last two equations and exchanging the order of summation, we obtain

$$
v_{k}=\sum_{l=0}^{\infty} y(l) q_{k}(l ; b)
$$

and

$$
w_{k}=\sum_{l=0}^{\infty} y(l) p_{k}(l ; b)
$$

where

$$
q_{k}(l ; b)=\sum_{r} h(r-2 k) \lambda_{r}(l ; b)
$$

and

$$
p_{k}(l ; b)=\sum_{r} g(r-2 k) \lambda_{r}(l ; b)
$$

By defining the time-reversed signal $x(l)=y(-l),(21)$ and (22) may be put in the form of convolution evaluated at the origin. Thus, letting

$$
\hat{v}_{k}(n)=\sum_{l=0}^{\infty} x(n-l) q_{k}(l ; b)
$$

and

$$
\hat{w}_{k}(n)=\sum_{l=0}^{\infty} x(n-l) p_{k}(l ; b)
$$

we have $v_{k}=\hat{v}_{k}(0)$ and $w_{k}=\hat{w}_{k}(0)$. Clearly, the $Z$ transforms of the sequences (25) and (26), respectively, are

$$
\hat{V}_{k}(z)=X(z) Q_{k}(z)
$$

and

$$
\hat{W}_{k}(z)=X(z) P_{k}(z) .
$$

By $Z$ transforming both sides of (23) and (24) with respect to the index $l$ and using recurrence (3), we obtain

$$
Q_{k}(z)=\sum_{r} h(r-2 k) \Lambda_{r}(z)=\Lambda_{0}(z) A(z)^{2 k} H\left(A(z)^{-1}\right)
$$

and

$$
P_{k}(z)=\Lambda_{0}(z) A(z)^{2 k} G\left(A(z)^{-1}\right)
$$

where the transfer functions

$$
H\left(A(z)^{-1}\right)=\sum_{r} h(r) A(z)^{r}
$$

and

$$
G\left(A(z)^{-1}\right)=\sum_{r} g(r) A(z)^{r}
$$

are frequency-warped versions of the original quadrature mirror filters. Similar transformations on transfer functions were introduced by Constantinides [11] for the design of IIR filters. However, in our case, the signal rather than the filter is frequency-warped.

From (25)-(30), it may be concluded that $v_{k}$ and $w_{k}$ correspond to the even indexed coefficients of the Laguerre expansion of the prefiltered signal, respectively, by the transfer functions (31) and (32). As a result of frequency warping, the frequency responses of the conventional QMF, which iare shown in Fig. 9(a), transform to those reported in Fig. 9(b). Notice that the new responses are still power complementary

$$
\left|H\left(e^{j \theta(\omega)}\right)\right|^{2}+\left|G\left(e^{j \theta(\omega)}\right)\right|^{2}=2 .
$$

Two equivalent structures implementing frequency-warped filter banks are shown in Fig. 10. In Fig. 10(a), the Laguerre expansion structure of Fig. 1 is cascaded with an ordinary analysis filter bank, whereas the diagram in Fig. 10(b) is obtained from (27) to (32). There, the ordinary downsamplers are replaced by the same structure as that shown in Fig. 2 with $A(z)^{2}$ in place of $A(z)$. This concept will be detailed in the next section. Convolving the warped signal with the time-reversed version of $h(n)$ [see Fig. 10(a)] is equivalent to 


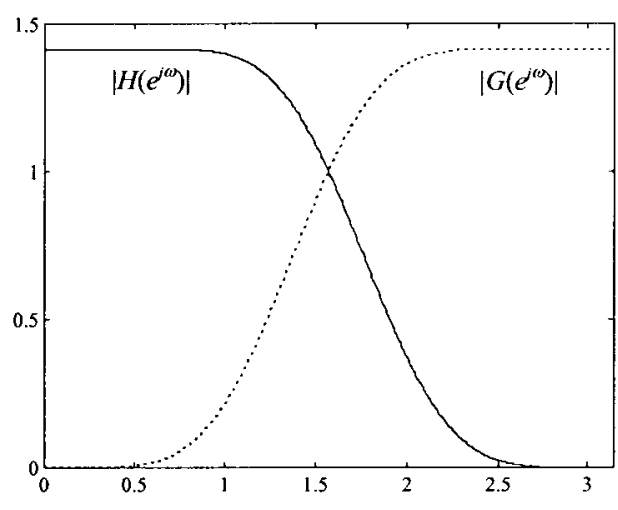

(a)

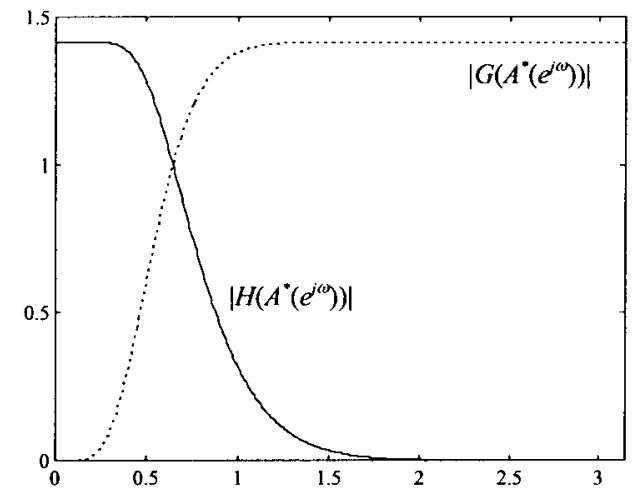

(b)

Fig. 9. Frequency responses of (a) ordinary quadrature mirror filters and (b) frequency-warped filter bank.

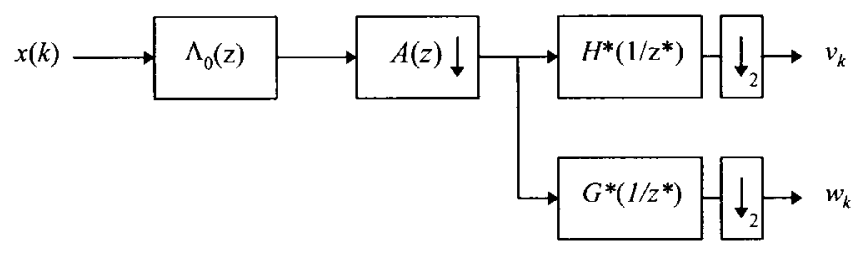

(a)

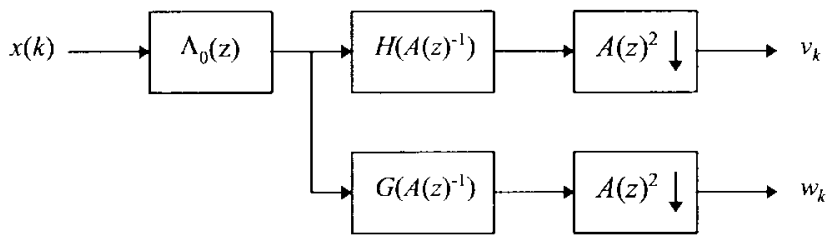

(b)

Fig. 10. Two equivalent structures implementing the frequency warping analysis filter bank. (a) Laguerre analysis cascaded by ordinary, critically sampled, analysis filter bank. (b) Embedded frequency warping analysis filter bank.

convolving the signal with a version of $h(n)$-warped in the opposite direction-and warping the result [see Fig. 10(b)]. The last operation is embedded in the generalized downsampler block $A(z)^{2} \downarrow$. The passband of $H\left(A(z)^{-1}\right)$ is wider or narrower than that of $H(z)$ if, respectively, $b$ is negative or positive. However, as a result of the final warping, the bandwidth of the output signal is at most the same as that of $H(z)$. Therefore, the downsampling factor 2, which is represented by the exponent of $A(z)$, is unchanged with respect to ordinary sampled filter banks.

Consider the synthesis filter bank shown in Fig. 11. The input-output relationship is

$$
u_{r}=\sum_{k} h(r-2 k) v_{k}+\sum_{k} g(r-2 k) w_{k} .
$$

Since it is a perfect reconstruction filter bank, the output recovers the Laguerre expansion coefficients $u_{r}$ of the signal $y(k)$. By substituting (33) in (12) and performing a simple change of the summation index, we obtain

$$
\begin{aligned}
Y(z)= & \left(H\left(A(z)^{-1}\right) \sum_{k} v_{k} A(z)^{2 k}\right. \\
& \left.+G\left(A(z)^{-1}\right) \sum_{k} w_{k} A(z)^{2 k}\right) \Lambda_{0}(z) .
\end{aligned}
$$

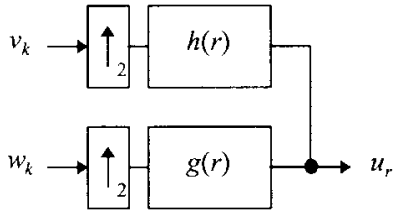

Fig. 11. Two-band critically sampled synthesis filter bank.

The last equation combines the inverse filter bank structure with the Laguerre expansion. Comparing this expression with (12), we observe that the signal is reconstructed by adding together the filtered even-order Laguerre components. This is a form of sampling theorem in the Laguerre domain. In fact, from (7), (19), (20) and exploiting the identity (15), it is possible to show that

$$
\begin{aligned}
V\left(A(z)^{-2}\right) & =\sum_{k} v_{k} A(z)^{2 k} \\
& =\frac{1}{2} H(A(z)) \Lambda_{0}(z)^{-1} Y(z)+\frac{1}{2} E_{H}(z)
\end{aligned}
$$

and

$$
\begin{aligned}
W\left(A(z)^{-2}\right) & =\sum_{k} w_{k} A(z)^{2 k} \\
& =\frac{1}{2} G(A(z)) \Lambda_{0}(z)^{-1} Y(z)+\frac{1}{2} E_{G}(z)
\end{aligned}
$$

where

$$
E_{H}(z)=H(-A(z)) \Lambda_{0}^{+}\left(-A(z)^{-1}\right) Y\left(A^{+}\left(-A(z)^{-1}\right)^{-1}\right)
$$

and

$$
E_{G}(z)=G(-A(z)) \Lambda_{0}^{+}\left(-A(z)^{-1}\right) Y\left(A^{+}\left(-A(z)^{-1}\right)^{-1}\right)
$$

are warped aliasing terms. By substituting (35) and (36) in (34), we conclude that the aliasing cancellation condition is

$$
\mathbf{T}\left(A(z)^{-1}\right) \tilde{\mathbf{T}}\left(A(z)^{-1}\right)=2 \mathbf{I}
$$

where $\mathbf{T}\left(A(z)^{-1}\right)$ is a warped version of the ac matrix (18). Since the map $z^{-1} \rightarrow A(z)$ is one-to-one, (37) and (17) are equivalent. 


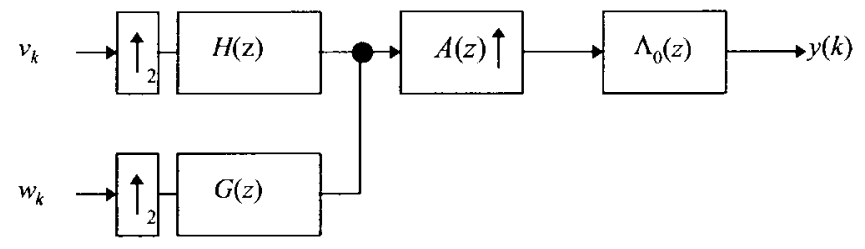

(a)

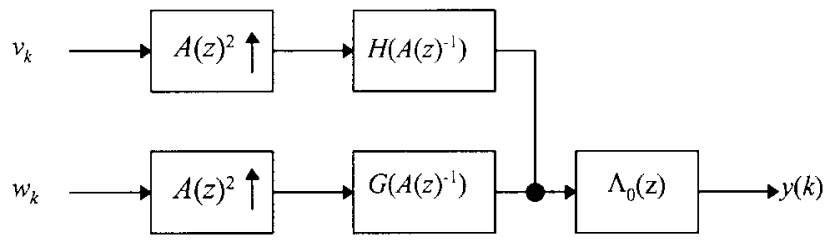

(b)

Fig. 12. Two equivalent structures implementing the frequency warping synthesis filter bank. (a) Laguerre synthesis cascaded by ordinary, critically sampled, synthesis filter bank. (b) Embedded frequency warping synthesis filter bank.

Two equivalent structures implementing the warped synthesis filter bank are shown in Fig. 12. In Fig. 12(a), the signal is reconstructed by means of a conventional filter bank cascaded with the Laguerre synthesis structure. The diagram in Fig. 12(b) is obtained from (34) and involves frequencywarped filter banks and generalized upsampling operators, as detailed in the next section.

The two-band orthogonal case is easily extended to $M$-band filter banks that are orthogonal or biorthogonal. It suffices to increase the number of filters from 2 to $M$, replacing $A(z)^{2}$ with $A(z)^{M}$ in all the expressions and diagrams. In biorthogonal structures, we need to consider two distinct pairs of analysis and synthesis filters. However, a single frequency warping does not lead to arbitrary bandwidth specification in the $M$-band case, when $M>2$. This is due to the fact that the warping parameter $b$ may be tuned in order to satisfy one of the cut-off requirements. The cut-off frequencies of the other bands follow the warping law given by the map $z^{-1} \rightarrow A(z)$.

\section{GENERALIZED DOWNSAMPLING AND UPSAMPLING}

In this section, we detail structures and properties of the generalized resampling operators $A(z)^{M} \downarrow$ and $A(z)^{M} \uparrow$. These blocks are included in the frequency-warped filter banks, as shown in the previous section.

Consider the diagram shown in Fig. 13(a), in which a timereversed signal $f(-k)$ is fed into a delay line composed of $M$-samples delay elements. At time $k=0$, the switches are closed toward the outputs. At this instant, the content of the delay line is exactly the $M$-fold downsampled causal part of $f(k)$. Thus, the structure in Fig. 13(a) is a noncausal implementation of the downsampling operation. Suppose we replace each unit delay element by a real first-order allpass filter $A(z)$, obtaining the structure shown in Fig. 13(b). The output samples

$$
f_{k}=\frac{1}{2 \pi} \int_{-\pi}^{\pi} d \omega F\left(e^{j \omega}\right) e^{j k M \theta(\omega)}
$$

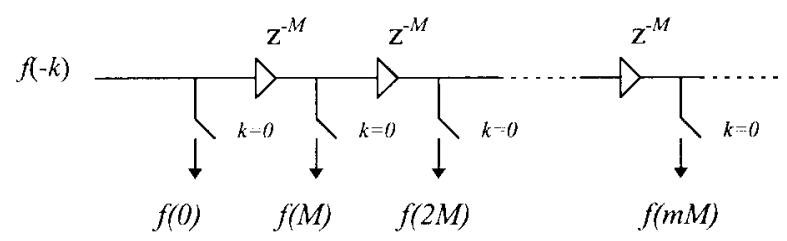

(a)

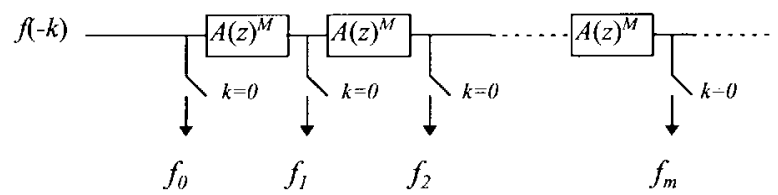

(b)

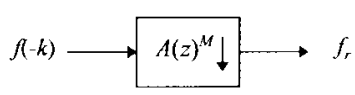

(c)

Fig. 13. Noncausal implementation of downsamplers. (a) Classical $M$-fold downsampler. (b) Generalized downsampler. (c) Its equivalent schematic symbol.

where $\theta(\omega)$ is given in (5), will no longer correspond to the samples of the input signal. Rather, each allpass filter introduces a frequency-dependent delay and, hence, a frequency-dependent sampling factor. For this reason, we denominate this structure as a generalized downsampler, denoting it with the symbol shown in Fig. 13(c), which is in agreement with Fig. 2(b). If the input is the complex sinusoid $f(k)=e^{j \omega_{0} k}$, the output of the generalized downsampler will be the sequence

$$
f_{k}=e^{j \omega_{0} k M \tau_{P}\left(\omega_{0}\right)}
$$

where $\tau_{P}(\omega)=\frac{\theta(\omega)}{\omega}$ is the phase delay of the allpass filter. Thus, the output is a resampled version of the input, with sample spacing $M \tau P\left(\omega_{0}\right)$. This quantity is frequencydependent and may happen to become less than one. In this case, the generalized downsampler is actually an interpolator, where the output rate is higher than that of the input. A necessary condition for that to be true is $b<0$.

The allpass filter $A(z)$ pertaining to the Laguerre domain filter bank downsampling structure has order one. In the simple case $b=0$, the allpass filters revert to unit delay elements, and the generalized downsampler is equivalent to the classical downsampler.

Consider now the structure shown in Fig. 14(a), where a unit pulse sequence is fed to an infinite tapped delay line. The weights of the line are altogether set by the input sequence $f_{r}$. The output of the line is

$$
y(k)=\sum_{r} f_{r} \delta(k-r M)
$$

i.e., the $M$-fold zero-inserted input sequence, whose $Z$ transform is

$$
Y(z)=F\left(z^{M}\right)
$$

By means of the same allpass transformation adopted for the downsamplers, we can generalize the upsampling operation, 


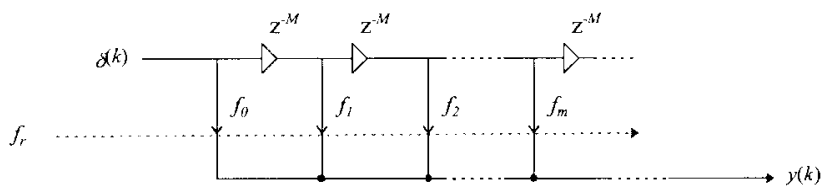

(a)

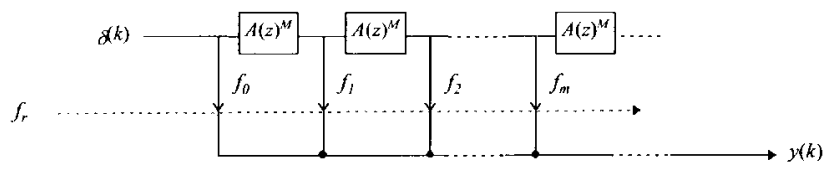

(b)

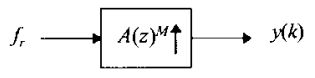

(c)

Fig. 14. Noncausal implementation of upsamplers. (a) Classical $M$-fold upsampler. (b) Generalized upsampler. (c) Its equivalent schematic symbol.

obtaining the diagram shown in Fig. 14(b). The $Z$ transform of the output of this structure is

$$
Y(z)=F\left(A(z)^{-M}\right)
$$

that is, a frequency-warped version of (38). For this reason, we define this structure to be a generalized upsampler and denote it with the symbol shown in Fig. 14(c). Except in trivial cases, the output of the generalized upsampler is not simply a zero-inserted version of the input since each input sample is multiplied by the impulse response of the filter corresponding to the respective power of $A(z)^{M}$.

\section{FREQUENCY WARPED WAVELETS}

\section{A. Laguerre Wavelets}

In the previous section, we showed how the computational structures associated with the Laguerre expansion and critically sampled filter banks may be merged, leading to a filter bank structure with frequency-warped transfer functions and generalized resampling. It is easy to extend this concept to iterated filter banks, including the ones associated with wavelet transform [14]-[16]. In fact, referring to (23) and (24), define the level 1 Laguerre scaling sequences and wavelets, respectively, as

$$
\begin{aligned}
& \phi_{1, k}(l)=\sum_{r} h(r-2 k) \lambda_{r}(l ; b) \\
& \psi_{1, k}(l)=\sum_{r} g(r-2 k) \lambda_{r}(l ; b) .
\end{aligned}
$$

We can write the single scale level wavelet expansion of the signal $y(l)$ as

$$
y(l)=\sum_{k}\left(v_{1, k} \phi_{1, k}(l)+w_{1, k} \psi_{1, k}(l)\right)
$$

where

$$
v_{1, k}=\sum_{l} y(l) \phi_{1, k}(l)
$$

and

$$
w_{1, k}=\sum_{l} y(l) \psi_{l, k}(l)
$$

respectively, are the level 1 Laguerre scaling and wavelet coefficients [see (21) and (22)]. Iterating the filter bank structure of Fig. 10 in a way similar to the usual construction of discrete wavelets [14]-[16], we can define the scaling and wavelet coefficients at higher scales, respectively, as

$$
v_{n+1, k}=\sum_{r} h(r-2 k) v_{n, r}
$$

and

$$
w_{n+1, k}=\sum_{r} g(r-2 k) v_{n, r}
$$

Accordingly, higher scale Laguerre scaling sequences and wavelets are defined, respectively, by the iterations

$$
\begin{aligned}
& \phi_{n+1, k}(l)=\sum_{r} h(r-2 k) \phi_{n, r}(l) \\
& \psi_{n+1, k}(l)=\sum_{r} g(r-2 k) \phi_{n, r}(l) .
\end{aligned}
$$

The $Z$-transform counterparts of (47) and (48), respectively, are

$$
\Phi_{n+1, k}(z)=\sum_{p} h(p) \Phi_{n, p+2 k}(z)
$$

and

$$
\Psi_{n+1, k}(z)=\sum_{p} g(p) \Phi_{n, p+2 k}(z) .
$$

From (29) and (30), in view of the correspondence between (40) with (23) and (41) with (24), we have

$$
\Phi_{1, k}(z)=\Lambda_{0}(z) A(z)^{2 k} H\left(A(z)^{-1}\right)=A(z)^{2 k} \Phi_{1,0}(z)
$$

and

$$
\Psi_{1, k}(z)=\Lambda_{0}(z) A(z)^{2 k} G\left(A(z)^{-1}\right)=A(z)^{2 k} \Psi_{1,0}(z) .
$$

From (49)-(52), iterating on the scale index $n$, we obtain

$$
\begin{aligned}
\Phi_{n, k}(z) & =A(z)^{2^{n} k} \Phi_{n, 0}(z) \\
\Psi_{n, k}(z) & =A(z)^{2^{n} k} \Psi_{n, 0}(z) \\
\Phi_{n+1,0}(z) & =H\left(A(z)^{-2^{n}}\right) \Phi_{n, 0}(z) \\
\Psi_{n+1,0}(z) & =G\left(A(z)^{-2^{n}}\right) \Phi_{n, 0}(z)
\end{aligned}
$$

from which

$$
\Phi_{n, 0}(z)=\Lambda_{0}(z) \prod_{r=0}^{n-1} H\left(A(z)^{-2^{r}}\right) .
$$

Comparing the $Z$ transforms of ordinary [14], [16] and Laguerre wavelets, we obtain the fundamental relationships

$$
\Phi_{n, k}(z)=\Lambda_{0}(z) \hat{\Phi}_{n, k}\left(A(z)^{-1}\right)=\Lambda_{2^{n} k}(z) \hat{\Phi}_{n, 0}\left(A(z)^{-1}\right)
$$


and

$$
\Psi_{n, k}(z)=\Lambda_{0}(z) \hat{\Psi}_{n, k}\left(A(z)^{-1}\right)=\Lambda_{2^{n} k}(z) \hat{\Psi}_{n, 0}\left(A(z)^{-1}\right)
$$

where $\Lambda_{2^{n} k}(z)$ is the $Z$ transform of the Laguerre sequence of order $2^{n} k$, as given in (2). $\hat{\Phi}_{n, k}(z)$ and $\hat{\Psi}_{n, k}(z)$, respectively, denote the $Z$ transform of ordinary scaling sequences and wavelets based on the same quadrature mirror filter pair $\{H, G\}$. This result is to be expected since the Laguerre wavelets revert to ordinary wavelets when the parameter $b=0$. In that case, $A(z)=z^{-1}$, and the Laguerre coefficients equal the input samples.

Notice from (56) that the Laguerre wavelets at lag $2^{n} k$ are obtained by convolving the lag 0 warped wavelet with the Laguerre sequence of order $2^{n} k$. Thus, at any fixed scale, there is a countable infinity of distinct Laguerre wavelets sharing the same magnitude frequency spectrum but differing in phase.

The Laguerre wavelets are frequency-warped and filtered versions of ordinary wavelets in the sense specified in Section II-B. This property is useful for building discrete wavelet transforms with nonoctave-spaced frequency bands. The Laguerre parameter $b$ controls the warping of the frequency axis: Compared with ordinary wavelets, positive values of $b$ produce narrower bands at high scale levels (low frequencies), whereas negative values of $b$ result in narrower bands at low scale levels (high frequencies). The cut-off frequencies of dyadic wavelets occurring at normalized frequencies of $\frac{\pi}{2^{n}}$ are mapped to the frequencies $\omega_{n}$, which are the roots of the transcendental equation $\theta\left(\omega_{n}\right)=\frac{\pi}{2^{n}}, n=1,2, \ldots$, where $\theta(\omega)$ is given in (5) (see also Fig. 7). Examples of frequency-warped wavelets and their frequency spectra are reported in Fig. 15, where they can be compared with ordinary wavelets. Positive values of the parameter $b$ lead to time-scale representations that are more selective at low frequencies than ordinary wavelets. This could be useful if it is known a priori that the signal energy is mostly concentrated in that region of the spectrum. On the other hand, negative values of $b$ lead to time-scale representations that are more selective at high frequencies than ordinary wavelets. This may be useful when the octaveband resolution is too poor to classify or detect characteristics of the signal. In coding problems, the parameter $b$ may be determined by optimizing a specific performance measure such as the coding gain of the transform.

As previously remarked, the filter $\Lambda_{0}(z)$ is needed for orthogonality and acts as a pre-emphasis filter, accounting for the peculiar shape of the frequency spectrum of warped wavelets. When $b>0$, this filter is lowpass, compensating for the energy loss due to the narrowing of the passbands at low frequencies. On the other hand, for $b<0$, the filter is highpass, compensating for the energy loss due to the narrowing of the passbands at high frequencies.

The time-domain expression of the Laguerre scaling sequences and wavelets can be obtained either from (40), (41), (47), (48), and the definition of ordinary wavelets or by inverting (55) and (56). We have

$$
\phi_{n, k}(m)=\sum_{r=0}^{\infty} \hat{\phi}_{n, k}(r) \lambda_{r}(m)
$$

and

$$
\psi_{n, k}(m)=\sum_{r=0}^{\infty} \hat{\psi}_{n, k}(r) \lambda_{r}(m) .
$$

Hence, the Laguerre wavelets are obtained in the form of a Laguerre expansion whose coefficients are the ordinary wavelets. In other words, the ordinary wavelets are the orthogonal projections of the warped wavelets onto the discrete Laguerre set.

Orthogonality and completeness of the Laguerre wavelets descend from the same properties of both the discrete Laguerre and ordinary wavelet sets. The expansion of any finite-energy signal in terms of the finite-scale frequency-warped wavelet basis (51) is formally identical to that of the ordinary discretetime wavelet transform

$$
y(k)=\sum_{m}\left(\sum_{n=1}^{N} w_{n, m} \psi_{n, m}(k)+v_{N, m} \phi_{N, m}(k)\right)
$$

where the expansion coefficients may be computed as in (43)-(46), and wavelets and scaling functions are given by (53) or (57) and (58).

The Laguerre wavelets realize an unconventional tiling of the time-frequency plane, shown in Fig. 16. There, the frequency-dependent group delay of each wavelet is plotted against the frequency axis. The horizontal lines correspond to the warped cut-off frequencies. Each cell of the plane corresponds to the time-frequency location of the corresponding wavelet. Clearly, the picture is schematic since the uncertainties of the wavelets may overlap several cells. The tiling characteristics with $b$ positive or negative are different, as can be seen by comparing Fig. 16(a) with Fig. 16(b). This is due to the fact that for positive $b$, the group delay decreases with frequency and, vice versa, for negative $b$, the delay increases. This behavior should be compared with that of ordinary wavelets, which are arranged in rectangular cells in the time-frequency plane.

The Laguerre wavelets have the same number of zero moments as their unwarped counterpart. It suffices to remark that to each factor $\left(1+z^{-1}\right)$ of $H(z)$ corresponds the following factor of $H\left(A(z)^{-1}\right)$

$$
1+A(z)=\frac{(1-b)\left(1+z^{-1}\right)}{1-b z^{-1}}=\sqrt{\frac{1-b}{1+b}} \Lambda_{0}(z)\left(1+z^{-1}\right)
$$

which, again, has the form of a bounded factor times $\left(1+z^{-1}\right)$. The regularity of the warped wavelets may be investigated with the same methods devised for ordinary wavelets [14], [17].

Our results, however, directly extend to continuous time, without any need for frequency renormalization, which is typical of ordinary wavelets. The recipe for continuous-time Laguerre wavelets is to expand the signal over a complete set of Laguerre functions $\left\{l_{r}(t)\right\}$ and then to expand the sequence of Laguerre coefficients onto discrete-time wavelets. In that 


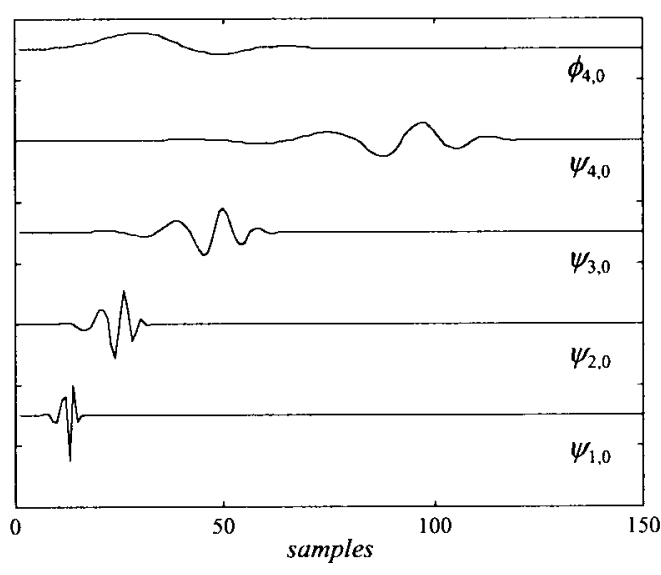

(a)

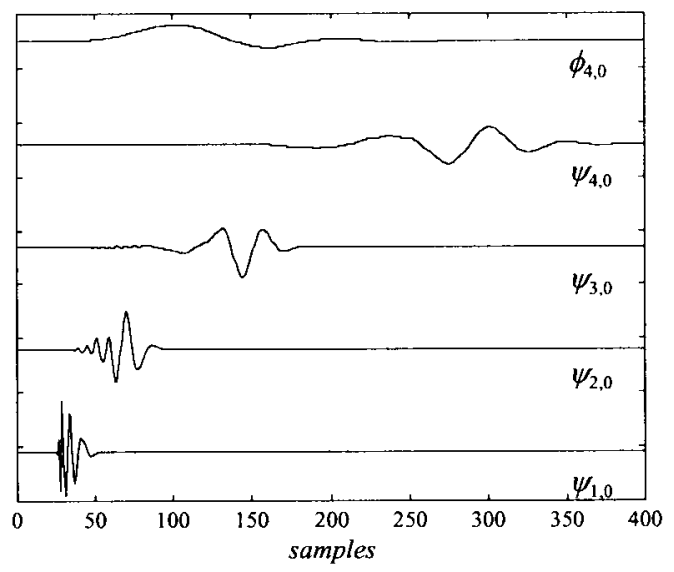

(b)

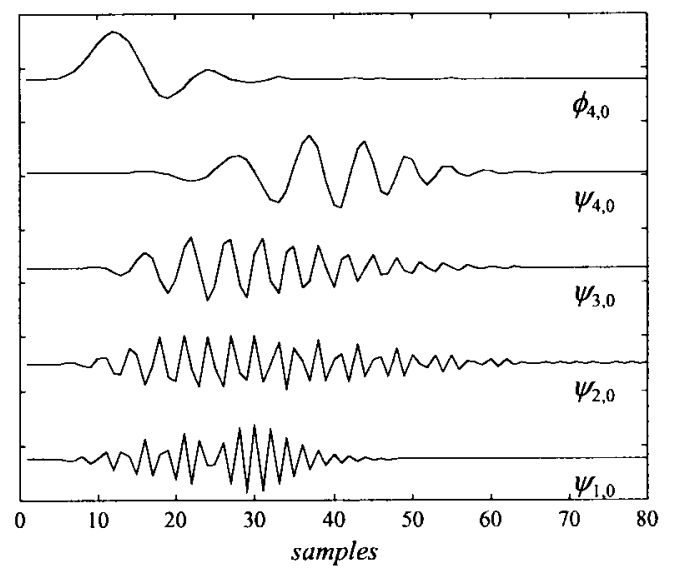

(c)

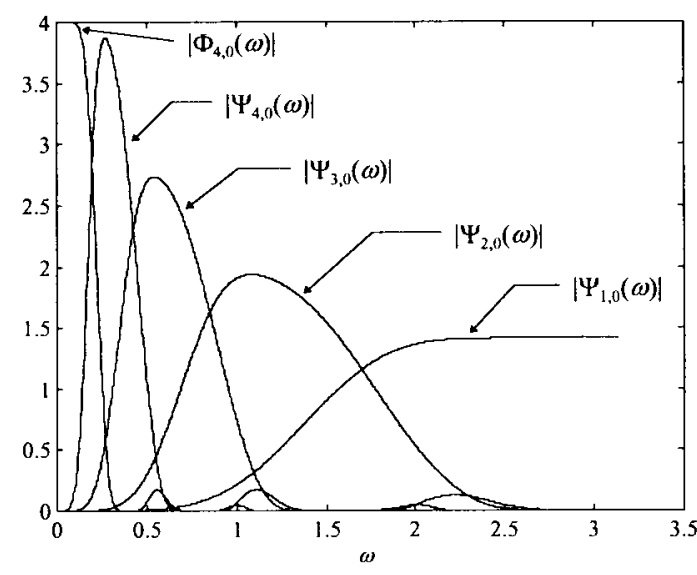

(d)

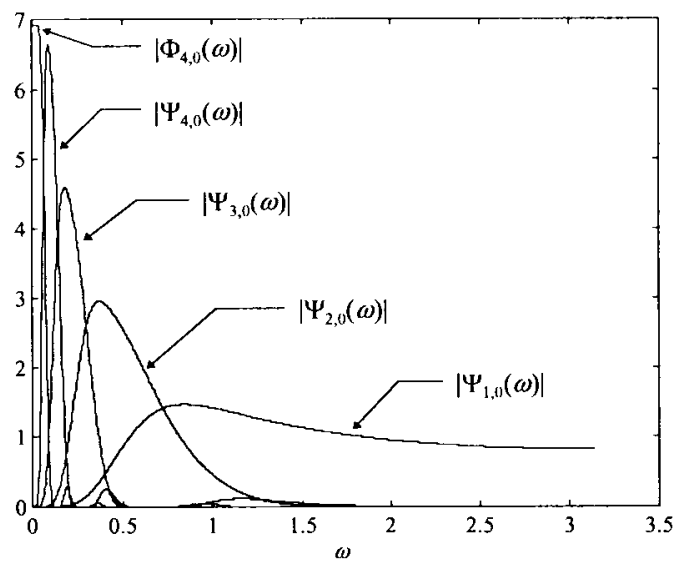

(e)

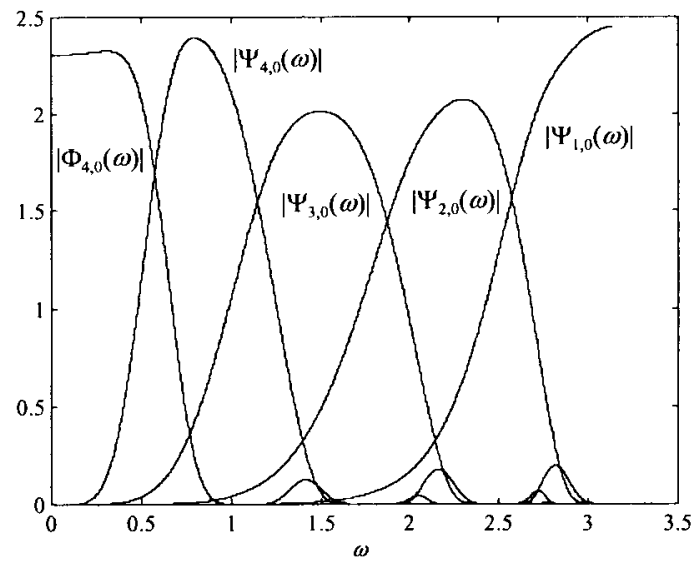

(f)

Fig. 15. Frequency warping wavelets and their frequency spectra. (a) Ordinary octave-band wavelets and scaling sequence. (b) frequency-warped wavelets with $b=0.5$. (c) With $b=-5$. The corresponding frequency spectra are shown in (d)-(f), respectively.

case, the continuous-time Laguerre wavelets are obtained in the form of a continuous-time Laguerre expansion whose coefficients are the ordinary discrete wavelets

$$
\psi_{n, k}(t)=\sum_{r=0}^{\infty} \hat{\psi}_{n, k}(r) l_{r}(t) .
$$

In fact, we can show [9] that the Laguerre sequences divided by the square root of the sampling rate approach the Laguerre functions as the sampling rate increases without bound, i.e., in this limit, (58) becomes (60). The continuous-time Laguerre functions interpolate the discrete ordinary wavelets to yield the continuous-time warped wavelets.

\section{B. Implementation}

The frequency-warped wavelet expansion may be computed in two formally equivalent ways. One method is to project the signal onto the Laguerre sequence basis first and then compute the ordinary wavelet transform of the Laguerre coefficients. This is achieved by feeding the signal into the iterated structure 


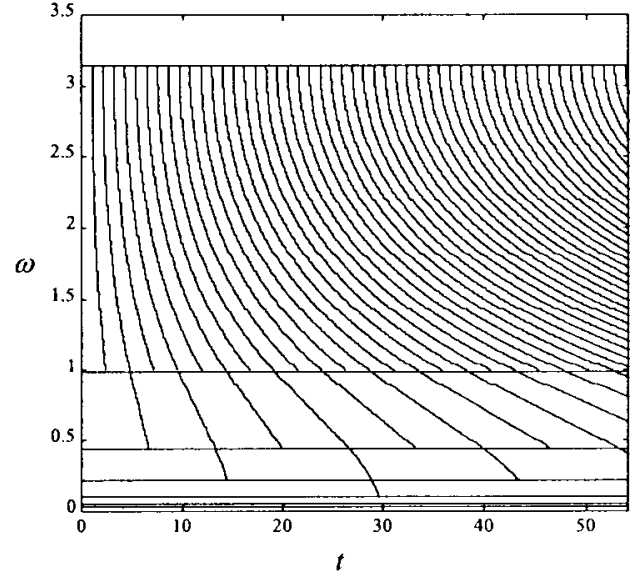

(a)

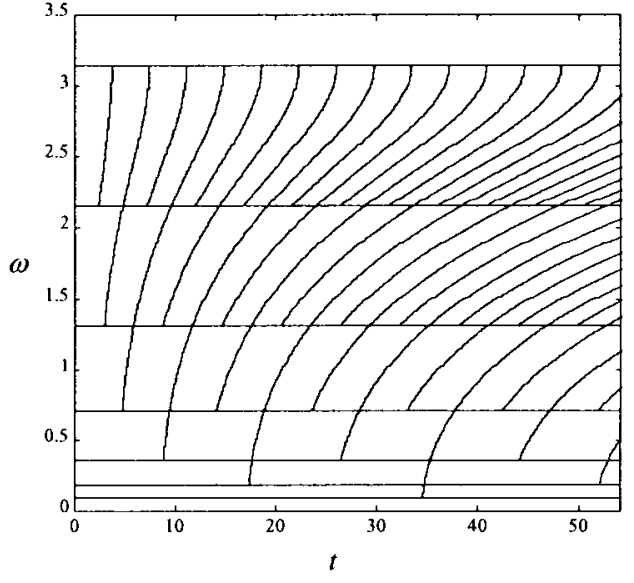

(b)

Fig. 16. Tiling the time-frequency plane with frequency-warped wavelets. (a) Corresponds to the Laguerre parameter $b=0.3$. (b) Corresponds to $b=-0.3$.

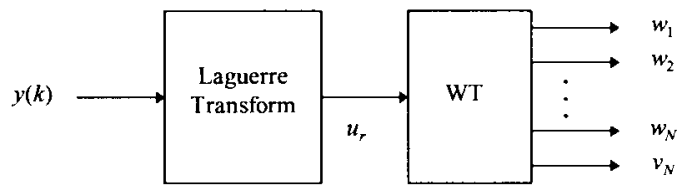

(a)

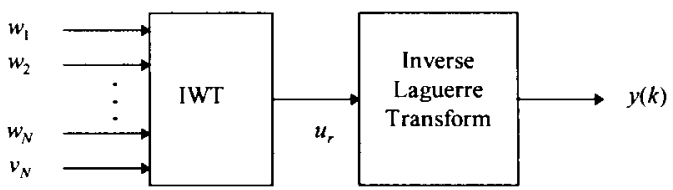

(b)

Fig. 17. Structure for computing the Laguerre wavelet transform. Implementation with Laguerre and wavelet transform blocks. (a) Analysis structure. (b) Synthesis structure.

shown in Figs. 1 and 2 and its output into a critically sampled iterated filter bank implementing the wavelet transform. The other method is to pass the signal through the orthogonal frequency-warped filter bank, including generalized downsampling, which is shown in Fig. 18(a). Similarly, there are two equivalent synthesis structures: one given by the ordinary wavelet synthesis filter bank followed by the Laguerre synthesis structure and the other given by the orthogonal frequencywarped synthesis filter bank shown in Fig. 18(b). This structure may be directly derived using (59) and recurrences (47) and (48) or, in the $Z$-transform domain, using (53) and the $Z$-transform of (59). From the discussion on generalized upsampling (downsampling) operators in Section IV, it follows that the synthesis (analysis) structure may be obtained by replacing the unit delay with the allpass filter $A(z)$ everywhere in the ordinary wavelet filter bank, including the upsampling (downsampling) operators, and prefiltering (postfiltering) with $\Lambda_{0}(z)$. It is worth noting that the generalized downsamplers in the frequency-warped filter bank are all located at the terminal nodes. Correspondingly, upsampling occurs only at input nodes of the synthesis structure. This is to be compared with the ordinary wavelet filter bank, in which downsampling or upsampling is distributed along the structure. The frequencywarped filter bank reverts to the ordinary wavelet filter bank when $A(z)=z^{-1}$. This is so by virtue of the "noble" identity [12] stating that $H\left(z^{2}\right)$ cascaded with twofold downsampling is equivalent to downsampling cascaded with $H(z)$. Hence, in this particular case, the terminal power-of-two downsamplers may be factored out in terms of twofold downsamplers that can be shifted back in between adjacent sections by repeatedly applying the "noble" identity. Unfortunately, an equivalent form of this identity is unavailable in the general frequencywarped case. In recent papers [18]-[20], one of the authors exploited allpass transformation in both the design of IIR wavelets and the construction of new transforms. In these cases, however, the allpass transformation was constrained to preserve the form of the ac matrix, requiring that $A(z)=$ $z^{-1} B\left(z^{2}\right)$, where $B(z)$ is arbitrary allpass. The first-order allpass transformation does not satisfy this property.

The complexity of the frequency-warped filter bank is generally greater compared with the cascade of Laguerre analysis (synthesis) and ordinary analysis (synthesis) wavelet filter bank. This is manly due to the generalized downsampling (upsampling) operators that are implemented by a long dispersive delay line. Sampling in the Laguerre domain requires a cascade of first-order allpass filters $A(z)$, each requiring two adds and two multiplies. The total number of warped wavelet coefficients required roughly equals the number $M$ of Laguerre coefficients needed to achieve the desired approximation of the signal. This number may be estimated from (10). It depends on the Laguerre parameter $b$ and is never smaller than the number $N$ of signal samples. The number of first-order allpass sections implementing generalized power-of-two downsampling does not depend on scale. In fact, at scale $n$, we need to compute roughly $2^{-2} M$ output samples, each requiring $2^{n}$ allpass sections, i.e., a total of $2 M$ multiplies per input sample at any scale level. The complexity of the warped filters grows with scale and is larger than that of ordinary QMF banks, even though lattice structures may be devised in which each unit delay is replaced by the required power of $A(z)$. The total cost is significantly reduced in special cases, e.g., when $b$ is a negative power of 2 . 


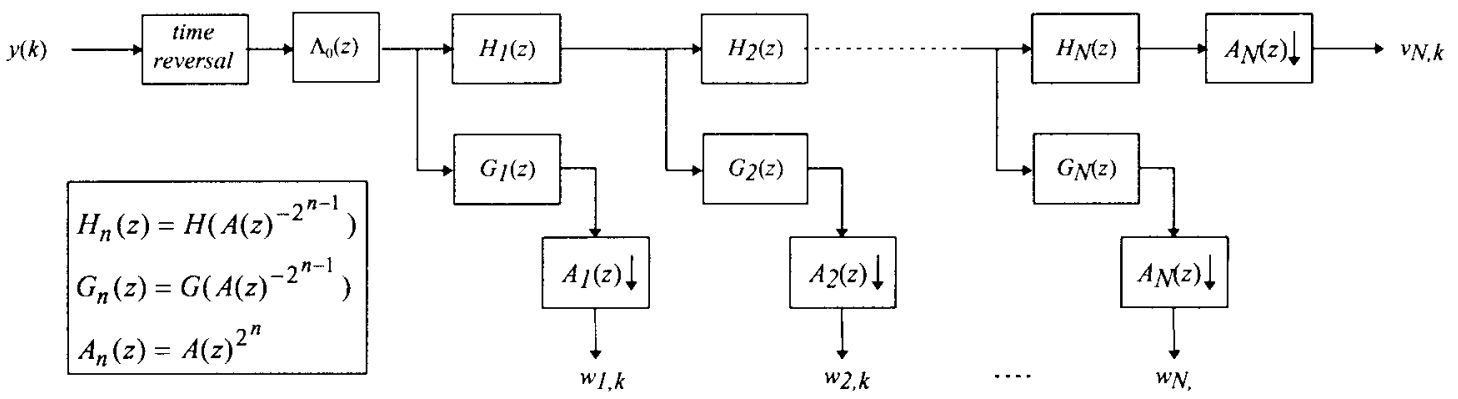

(a)

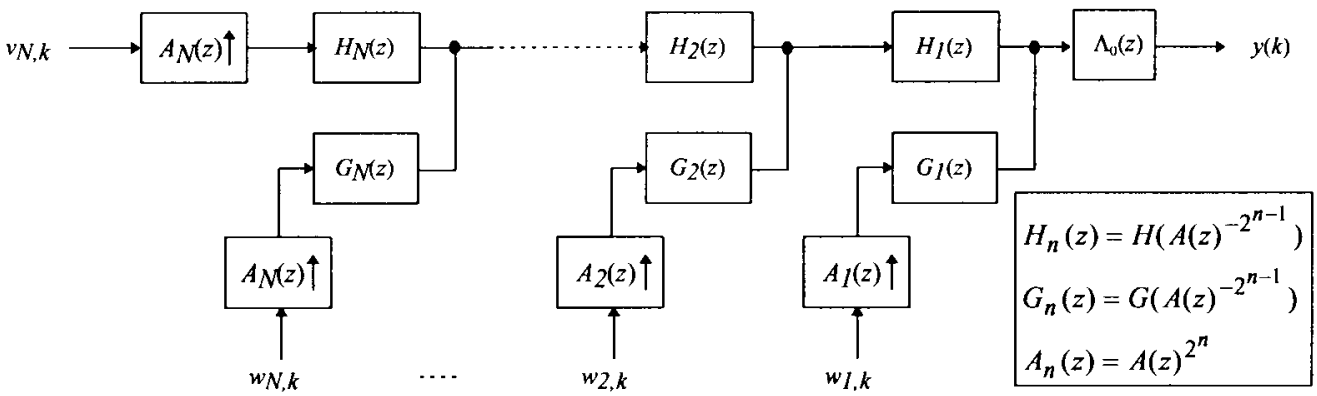

(b)

Fig. 18. Frequency-warped wavelet transform. (a) Analysis. (b) Synthesis structure with embedded warping.

The cost of the structure in Fig. 17 equals the cost of Laguerre transform of $N$ input samples plus the cost of wavelet transform of $M$ input samples. The former is implemented in a length- $M$ dispersive delay line, requiring $2 M$ multiplies and adds per input sample, whereas the cost of the orthogonal wavelet filter bank with degree $L$ FIR filters is approximately $2(L+3) M$ multiplies and $2(L+1) M$ adds, regardless of the number of scale levels. The total cost is therefore $2 M(N+$ $L+3)$ multiplies and $2 M(N+L+1)$ adds. With current technologies, this structure is more efficient than the filter bank with embedded warping. However, the latter has a great theoretical relevance, showing that the frequency-warped wavelet transform may be obtained by warping the wavelets rather than the signal, provided the sampling operators are generalized.

\section{CONCLUSION}

In this paper, we have demonstrated the existence of discrete-time orthogonal wavelets with nonoctave spaced frequency bands and frequency-dependent sampling rates. The construction of the new bases involves Laguerre expansion and sampling in the Laguerre domain. The discrete-time bases may be extended to continuous-time via continuous-time Laguerre expansions.

By varying a single parameter, we can design wavelets with desired bandwidth at a given scale. Hence, the new bases can be adapted to signals and allow us to perform higher resolution analysis in regions of interest of the frequency spectrum.

The computational structures for the warped wavelet transform are more complex than the conventional iterated filter bank. We have suggested and compared two alternative schemes. Both of them are noncausal, and we can compute only a finite number of coefficients pertaining to a finite duration input. We have shown that the Laguerre transform cascaded with the ordinary wavelet transform is a more efficient computational structure than filter banks with embedded warping.

Potential applications of the new bases are in off-line coding, signal analysis and feature extraction, transient detection, and system identification and control.

\section{REFERENCES}

[1] T. Oliveira e Silva, "On the determination of the optimal pole position of Laguerre filters," IEEE Trans. Signal Processing, vol. 43, pp. 2079-2087, Sept. 1995.

[2] _ " "Optimality conditions for truncated Laguerre networks," IEEE Trans. Signal Processing, vol. 42, pp. 2528-2530, Sept. 1994.

[3] C. Braccini and A. V. Oppenheim, "Unequal bandwidth spectral analysis using digital frequency warping," IEEE Trans. Acoust., Speech, Signal Processing, vol. ASSP-22, pp. 236-244, Aug. 1974.

[4] A. V. Oppenheim and D. H. Johnson, "Discrete representation of signals," Proc. IEEE, vol. 60, pp. 681-691, June 1972.

[5] A. V. Oppenheim, D. H. Johnson, and K. Steiglitz, "Computation of spectra with unequal resolution using the FFT," Proc. IEEE, vol. 59, pp. 299-301, Feb. 1971.

[6] R. G. Baraniuk and D. L. Jones, "Warped wavelets bases: Unitary equivalence and signal processing," in Proc. IEEE Int. Conf. Acoust., Speech, Signal Process.-ICASSP, 1993, vol. III, pp. 320-323.

[7] -Unitary equivalence: A new twist on signal processing," IEEE Trans. Signal Processing, vol. 43, pp. 2269-2282, Oct. 1995.

[8] M. J. Gottlieb, "Concerning some polynomials orthogonal on a finite or enumerable set of points," Amer. J. Math., vol. 60, pp. 453-458, 1938.

[9] P. W. Broome, "Discrete orthonormal sequences," J. Assoc. Comput. Machinery, vol. 12, no. 2, pp. 151-168, 1965.

[10] M. A. Masnadi-Shirazi, "Optimum Laguerre networks for a class of discrete-time systems," IEEE Trans. Signal Processing, vol. 39, pp. 2104-2108, Sept. 1991.

[11] A. G. Constantinides, "Spectral transformations for digital filters," Proc. IEEE, vol. 58, pp. 1585-1590, Aug. 1970.

[12] P. P. Vaidyanathan, Multirate Systems and Filter Banks. Englewood Cliffs, NJ: Prentice-Hall, 1993.

[13] M. Vetterli and J. Kovacevic, Wavelets and Subband Coding. Englewood Cliffs, NJ: Prentice-Hall, 1995.

[14] I. Daubechies, Ten Lectures on Wavelets. Philadelphia, PA: SIAM, 1992. 
[15] S. Mallat, "A theory for multiresolution signal decomposition: The wavelet representation," IEEE Trans. Pattern Anal. Machine Intell., vol. 11, pp. 674-693, July 1989.

[16] G. Evangelista, "Orthonormal wavelet transforms and filter banks," in Proc. IEEE 23rd Asilomar Conf., Pacific Grove, CA, 1989.

[17] O. Rioul, "A discrete-time multiresolution theory," IEEE Trans. Signal Processing, vol. 41, pp. 2591-2606, Aug. 1993.

[18] G. Evangelista, "Wavelet transforms and wave digital filters," in Wavelets and Applications, Y. Meyer, Ed. New York: Springer-Verlag, 1992, pp. 396-412.

[19] _ "Comb and multiplexed wavelet transforms and their applications to signal processing," IEEE Trans. Signal Processing, vol. 42, pp. 292-303, Feb. 1994.

[20] _ "Pitch synchronous wavelet representations of speech and music signals," IEEE Trans. Signal Processing, Special Issue on Wavelets and Signal Processing, vol. 41, pp. 3313-3330, Dec. 1993.

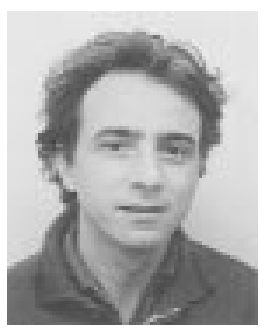

Gianpaolo Evangelista (M'92) received the laurea in physics, summa cum laude, from the University of Napoli "Federico II," Naples, Naples, Italy, in 1984 and the M.Sc. and Ph.D. degrees in electrical engineering from the University of California, Irvine, in 1987 and 1990, respectively.

$\mathrm{He}$ is a Researcher at the Department of Physical Sciences, University of Napoli "Federico II." From 1985 to 1986, he was a researcher at the Centre d'Etudes de Mathematique et Acoustique Musicale (CEMAMU/CNET), Paris, France, where he contributed to the development of the UPIC system for interactive sound synthesis from graphic input, and from 1991 to 1994, he was a Research Engineer at the Microgravity Advanced Research and Support Center, Naples, where he developed algorithms for object tracking and image velocimetry applied to fluid motion analysis. His current interests include signal and image processing; time-frequency representations; wavelets; and analysis, synthesis and coding of speech and music.

Dr. Evangelista was a recipient of the Fulbright fellowship.

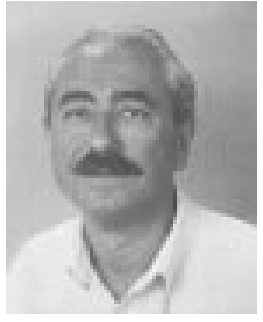

Sergio Cavaliere received the laurea in electronic engeneering, summa cum laude, from the University of Napoli "Federico II," Naples, Italy, in 1971.

From 1972 to 1973 , he was with the Department of Mathematics, CNR, University of Siena, performing research on logic, computability, and recursiveness. Since 1974, he has been a Research Associate with the Department of Physical Sciences, University of Napoli "Federico II." In 1986, he spent an academic year at the Media Laboratory, Massachussets Institute of Technology, Cambridge. From 1987 to 1991, he received a research grant for a project devoted to the design of VLSI chips for real-time sound processing and for the realization of the musical audio research station (MARS) workstation for sound manipulation at IRIS, Rome, Italy. Currently, he is also a Research Associate with INFN for the realization of very-large systems for data acquisition from nuclear physics experiments and for the development of techniques for the detection of signals in high-level noise. His interests include sound and music signal processing, signal transforms and representations, VLSI, and specialized computers for sound manipulation. 